\title{
Numerical Analysis on the Stability of Layered Surrounding Rock Tunnel Under the Conditions of Different Inclination Angle and Thickness
}

\author{
Wang Jingyuan*, Deng Xianghui, Cao Weiping \\ School of Civil Engineering, Xi' an Technological University, Xi' an, China \\ Email address: \\ 15615557600@163.com (Wang Jingyuan), xianghuideng@xatu.edu.cn (Deng Xianghui), 1920476215@qq.com (Cao Weiping) \\ *Corresponding Author
}

To cite this article:

Wang Jingyuan, Deng Xianghui, Cao Weiping. Numerical Analysis on the Stability of Layered Surrounding Rock Tunnel Under the Conditions of Different Inclination Angle and Thickness. American Journal of Traffic and Transportation Engineering.

Vol. 4, No. 2, 2019, pp. 67-74. doi: 10.11648/j.ajtte.20190402.14

Received: March 26, 2019; Accepted: April 27, 2019; Published: May 31, 2019

\begin{abstract}
To study the relationship between the stability of layered surrounding rock tunnel and the thickness and inclination angle of the layered rock, using the discrete element analysis software UDEC, the deformation and stress characteristics of layered surrounding rock tunnel are analyzed under different inclination angle and thickness conditions after the tunnel excavation. Meanwhile, the influence of different inclination angle and thickness on the stability of layered surrounding rock tunnel is studied. The results show that when the layer thickness and the excavation span are the same, the vertical displacement, horizontal displacement and maximum principal stress of the surrounding rock of the tunnel show a low of increasing first and then decreasing with the increase of the inclination angle of the structural plane. When the inclination angle and excavation span are same, the vertical displacement, horizontal displacement and maximum principal stress of layered surrounding rock of the tunnel gradually decrease with the increase of the layer thickness. When the inclination angle of the structural plane is $30^{\circ}$, the vertical displacement of the surrounding rock of the layered surrounding rock tunnel is the largest; When the inclination angle of the structural plane is $45^{\circ}$, the horizontal displacement of the surrounding rock of the layered surrounding rock tunnel is the largest; When the inclination angle of the structural plane is $45^{\circ}$, the layered surrounding rock tunnel principal stress is the largest.
\end{abstract}

Keywords: Layered Surrounding Rock Tunnel, Inclination Angle, Thickness, Stability

\section{Introduction}

With the continuous extension of the transportation system to the mountains, more and more tunnels engineering are crossing the layered rock mass. Due to the influence of rock thickness and inclination angle of joint surface and structural plane, there are often engineering accidents such as bedding slip, large deflection under eccentric pressure, support damage and even overall damage and instability of the tunnel [1-3].

For the layered surrounding rock tunnel, the numerical simulation method is often used to build the structural models by simplifying the geological conditions, load conditions, boundary conditions and initial stress field. Through the rapid calculation of the computer, the numerical solution that meets the needs of engineering can be obtained, which can better reflect the actual law. Meanwhile, it can be used as an alternative to expensive and complex large-scale field tests to effectively solve various complex engineering problems [4-7]. Zhao used the discrete element UDEC software to study the stability of tunnel surrounding rockinclination with different inclination angle of rock layers. When the inclinationinclinationangle was small, the vault was prone to bending failure [8]. Ou used the jointed constitutive models provided by FLAC to reasonably simulate the anisotropic characteristics of layered rock mass failure by taking into account the composite types of rock mass failure 
and bedding failure then analyzed the stability of layered rock mass slope [9]. Song used the three-dimensional discrete element method (3DEC) to explore the influence of structural surface distribution characteristics on the deformation of tunnel surrounding rock, then summarized two conditions of the influence of structural plane line density on tunnel deformation. When $\lambda \leq 0.2$, the bending deformation of the surrounding rock was greater than the shear deformation along the structural plane, which was a stress-type large deformation. When $0.2<\lambda \leq 0.4$, the shear deformation along the structural plane was greater than the bending deformation of the surrounding rock, which was large deformation of structures [10]. Based on the Drucker-Prager criterion, Wang established an anisotropic mechanical model of layered rock mass by considering the mechanical properties of the structural plane and bedrock respectively, then applied the model to the calculation and analysis of layered rock mass of underground engineering. The results can better reflect the anisotropy of layered rock mass [11]. Wang revealed the failure mode and regularity of the gently inclined rock slope of the Qijiang River in Chongqing, then concluded that the main inducement of rock cavity failure is pressure [12]. Based on engineering background of the return ailway in a coal mine, Wang used the finite difference software FLAC3D to simulate the deformation and failure of layered composite surrounding rock roadway before and after support. Meanwhile, the corresponding bolt-shotcrete support form was proposed by the composite double beam coupling mechanism and numerical simulation results of tunnel surrounding rock [13].

At present, there are few researches on the stability of layered surrounding rock tunnels, and most of them are analyzed for single factors. Therefore, the process and characteristics of tunnel deformation and instability of layered surrounding rock under the conditions of different inclinationinclination angles and thickness of layered rock mass structural plane. In this way, the deformation, stability and damage characteristics of surrounding rock of layered surrounding rock tunnel can be more comprehensively reflected.

\section{Numerical Model and Parameter Selection}

\subsection{Calculation Model and Boundary Conditions}

The numerical simulation was carried out by using the discrete element analysis software UDEC to analyze the steady state of the hole without the support structure after excavation. The tunnel excavation section is straight wall semi-circular arch. The full-section excavation method is used in tunnel construction.

According to the Saint-Venant's principle in elastic mechanics, after tunnel excavation, the corresponding stress redistribution only occurs within a certain range close to the cavern. According to the current research, when the tunnel excavation range exceeds 5 times the holes diameter, it can be considered that the excavation has less influence on the surrounding rock. Therefore, the model size in the horizontal direction is 5 times the span distance of the hole. In the vertical direction, the value can be taken according to the actual situation of the project.Meanwhile, in order to reduce the number of calculation units and simplify the calculation, a part of the thickness of the formation is added during the modeling, and the remaining part of the formation is converted into an equivalent load to be loaded on the upper part of the model.

The displacement boundary conditions are as follows: the lower boundary is a fixed boundary, the $\mathrm{X}$ and $\mathrm{Y}$ directions are zero, the horizontal constraint is applied in the left and right direction, and the X-direction displacement is zero. The mechanical model established in this paper is a plane strain model. In order to avoid the displacement of the model perpendicular to the plane direction, the stress $\sigma_{\mathrm{zz}}$ in the front and back directions is applied to the model.

\subsection{Rock Mass and Structural Plane Parameters}

The layered surrounding rock is a sedimentary rock with mudstone and sandstone. Therefore, the sandstone is selected as the research object. According to The Code for Design of Road Tunnel and the Handbook of Engineering Geology, the relevant physical and mechanical parameters of sandstone are taken [14]. The results are shown in Tables 1 and 2.

Table 1. The value of mechanical parameters of rock mass.

\begin{tabular}{llllll}
\hline Material & Elastic Modulus $\boldsymbol{E} / \mathbf{G P a}$ & Poisson ratio $\boldsymbol{\mu}$ & Bulk density $\boldsymbol{\gamma} / \mathbf{k N} \cdot \mathbf{m}^{-\mathbf{3}}$ & $\mathbf{C o h e s i o n} \boldsymbol{C} / \mathbf{k P a}$ & Friction $\left.\boldsymbol{\Phi} / \mathbf{(}^{\mathbf{}}\right)$ \\
\hline Sandstone & 10.0 & 0.2 & 24.0 & 800.0 & 38.0 \\
\hline
\end{tabular}

Table 2. The value of mechanical parameters of structural plane.

\begin{tabular}{lllll}
\hline Material & Normal stiffness $\boldsymbol{K}_{n} / \mathbf{G P a}$ & Shear stiffness $\boldsymbol{K}_{s} / \mathbf{G P a}$ & Friction angle $\Phi /\left(^{0}\right)$ & $\mathbf{C o h e s i o n} \boldsymbol{C} / \mathbf{k P a}$ \\
\hline Sandstone joint & 4.0 & 2.0 & 24.0 & 100.0 \\
\hline
\end{tabular}

In the numerical simulation calculation, the bulk modulus $\mathrm{K}$ and the shear modulus $\mathrm{G}$ of the rock mass need to be determined, and the calculation formulas are respectively:

$$
K=\frac{E}{3(1-2 \mu)}
$$

$$
G=\frac{E}{2(1+\mu)}
$$

According to the elastic modulus E and Poisson's ratio $\mu$ of the sandstone in Table 1, the parameters $\mathrm{K}$ are $5.6 \times 10^{3} \mathrm{MPa}$ and $\mathrm{G}$ are $4.2 \times 10^{3} \mathrm{MPa}$. 


\subsection{Calculation Condition}

Because the inclinationinclination angle and layer thickness of the rock are important factors affecting the self-stability of the layered surrounding rock [15], the inclinationinclination angle and layer thickness of the rock are assumed respectively. From theinclination inclination angle of the rock in nature, various inclination angles are available. In order to make the study not lose its generality, the inclination angles of the rock layers are assumed to be $0^{\circ}$, $15^{\circ}, 30^{\circ}, 45^{\circ}, 60^{\circ}, 75^{\circ}$ and $90^{\circ}$ respectively. According to the current classification of layered rock mass, the layer thicknesses are $1.20 \mathrm{~m}, 0.75 \mathrm{~m}, 0.30 \mathrm{~m}$ and $0.10 \mathrm{~m}$, respectively, representing huge thick layers, thick layers, medium thick layers and thin layers. Considering that there are many cases of one-way two-lane road tunnels, the calculated excavation span is $12.0 \mathrm{~m}$. Therefore, for the different inclination angles and layer thicknesses of layered surrounding rock, a total of $28(7 \times 4)$ models needs to be established. The typical calculation models are shown in Figure 1.

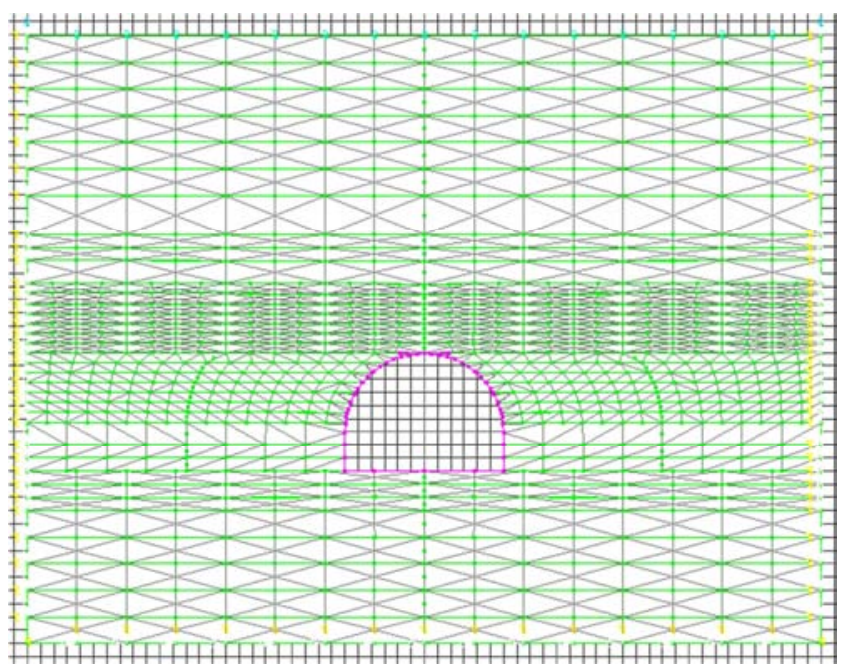

(a) layer thickness $0.75 \mathrm{~m}$, inclination angle $0^{\circ}$

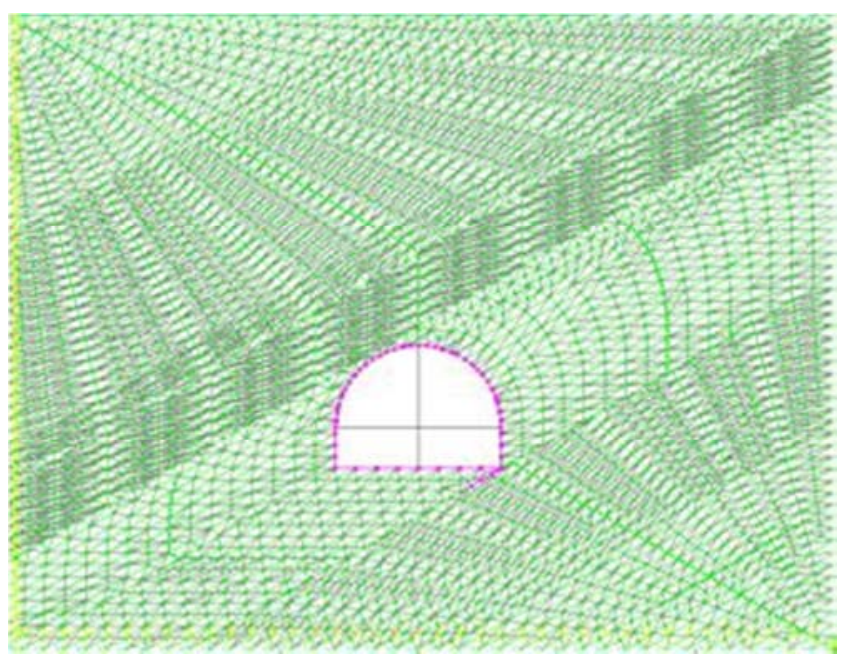

(b) layer thickness $0.75 \mathrm{~m}$, inclination angle $30^{\circ}$

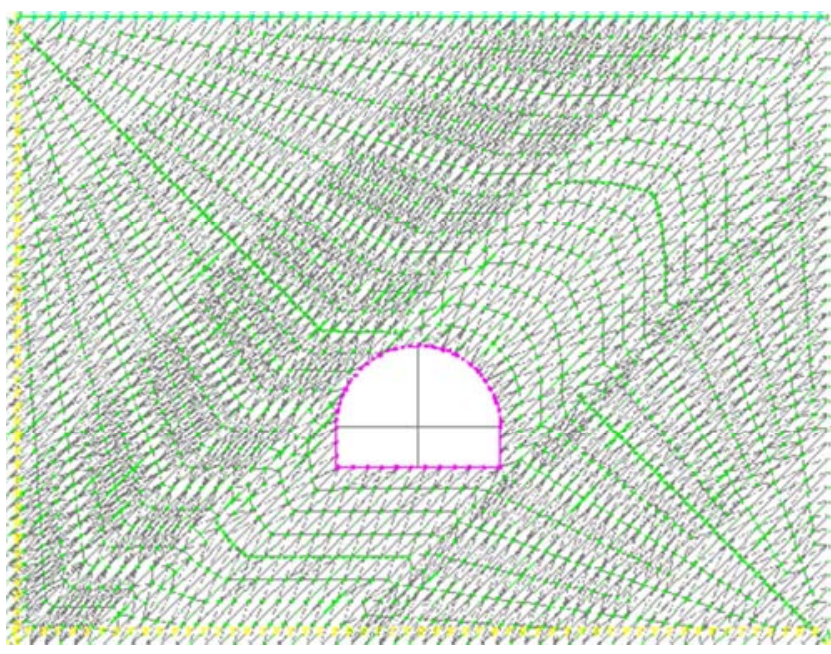

(c) layer thickness $0.75 \mathrm{~m}$, inclination angle $45^{\circ}$

Figure 1. Numerical simulation models.

\section{Numerical Simulation Results Analysis}

The above mentioned 28 models are analyzed and the results are obtained after cyclic iterative calculation. In order to make the calculation more effective, the result is processed by post-processing software TECPLOT.

\subsection{Displacement Analysis}

\subsubsection{Vertical Displacement Analysis}

According to the previous analysis, numerical simulations were carried out on the inclination angles of $0^{\circ}, 15^{\circ}, 30^{\circ}, 45^{\circ}$, $60^{\circ}, 75^{\circ}$ and $90^{\circ}$ as well as thicknesses of $0.10 \mathrm{~m}, 0.30 \mathrm{~m}$, $0.75 \mathrm{~m}$ and $1.20 \mathrm{~m}$ respectively. The maximum vertical displacement calculation results are shown in Figure 2.

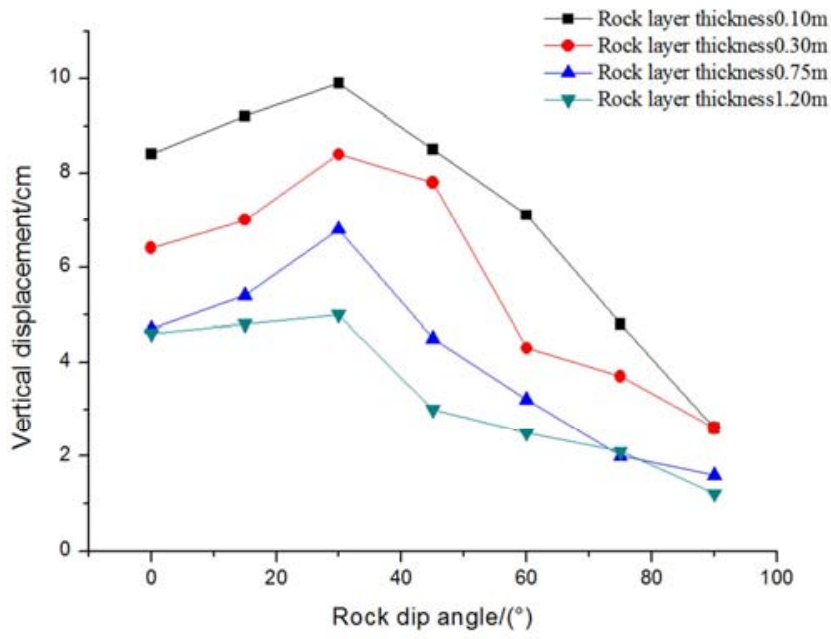

Figure 2. The maximum vertical displacement corresponding to the different inclinations.

As can be seen from Figure 2, when the inclination angle of the structural plane is between $0^{\circ}$ and $30^{\circ}$, the vertical displacement of the surrounding rock of the layered surrounding rock tunnel increases with the increase of the inclination angle of the structural plane. When the inclination 
angle is $30^{\circ}$, the vertical displacement of surrounding rock of the layered surrounding rock tunnel appears the maximum value, which is $9.9 \mathrm{~cm}$. When the inclination angle of the structural plane is between $30^{\circ}$ and $90^{\circ}$, the vertical displacement of the surrounding rock of the layered surrounding rock tunnel decreases with the increase of the inclination angle of the structural plane. When the structural plane inclination angle is $90^{\circ}$, the maximum vertical displacement of the surrounding rock of the layered surrounding rock tunnel is reduced to $1.2 \mathrm{~cm}$. Therefore, the vertical displacement of the surrounding rock of the layered surrounding rock tunnel are increasing first and then decreasing with the increase of the inclination angle of the structural plane. At the same time, the thickness of the rock layer and the vertical displacement of the surrounding rock also show a strong correlation. When the thickness of rock layer is $0.10 \mathrm{~m}$, the maximum vertical displacement of the surrounding rock after tunnel excavation is $9.9 \mathrm{~cm}$. When the thickness of rock layer is $0.30 \mathrm{~m}$, the maximum vertical displacement of surrounding rock after tunnel excavation is $8.4 \mathrm{~cm}$. When the thickness of rock layer is $0.75 \mathrm{~m}$, the maximum vertical displacement of surrounding rock after tunnel excavation is $6.8 \mathrm{~cm}$. When it is $1.20 \mathrm{~m}$, the maximum vertical displacement of surrounding rock after tunnel excavation is only $5.0 \mathrm{~cm}$, which is $50.5 \%$ of the maximum vertical displacement of the $0.10 \mathrm{~m}$ layer surrounding rock. Therefore, with the increase of rock layer thickness, the vertical displacement of surrounding rock after tunnel excavation appears to gradually decrease.

From the calculation process, the position of the maximum vertical displacement of the rock layers with different inclination angles also shows a certain regularity. In order to reflect the variation of vertical displacement of different inclination angles, this paper describes the rock layer thickness of $0.75 \mathrm{~m}$ and the inclination angle of $0^{\circ}, 30^{\circ}$ and $90^{\circ}$. The vertical displacement cloud diagrams of different inclination angles are shown in Figure 3.

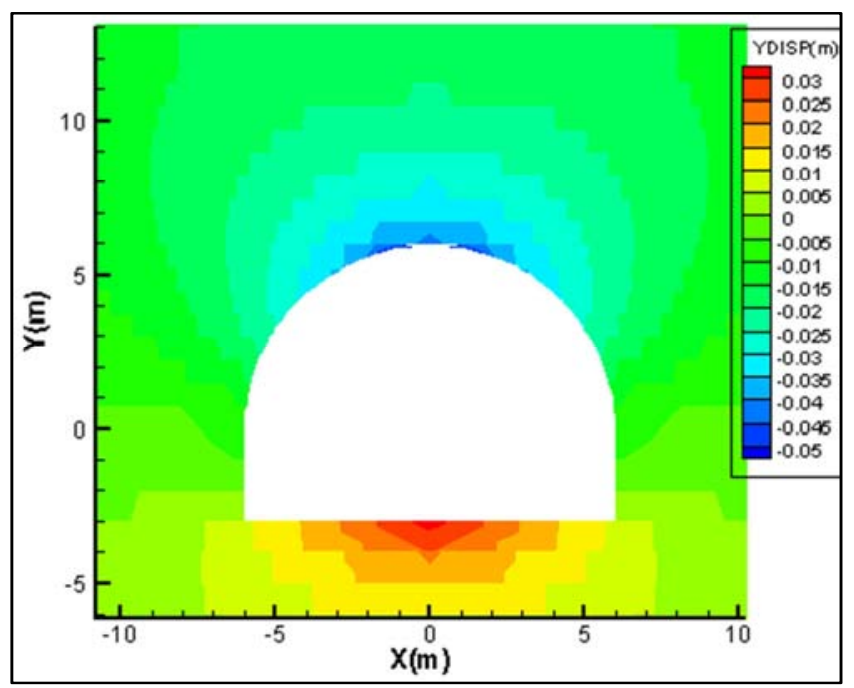

(a) Vertical displacement diagram with inclination $0^{\circ}$

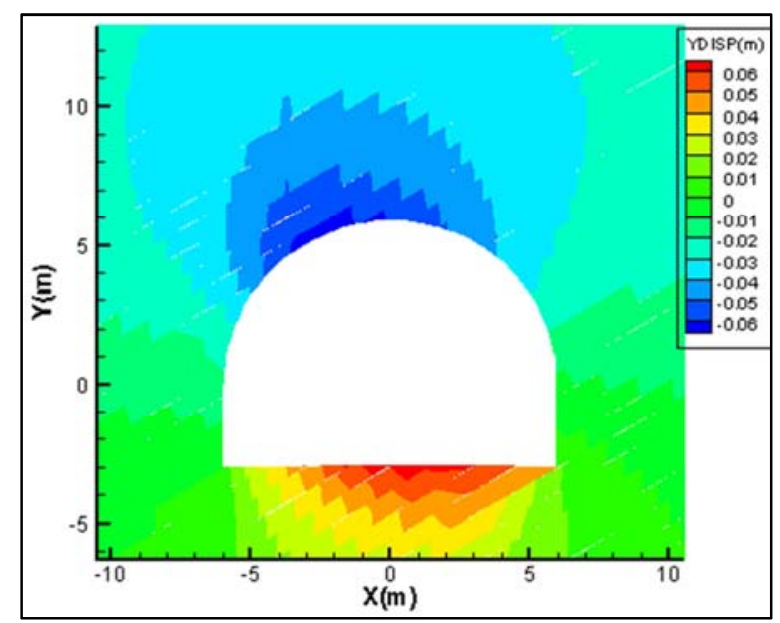

(b) Vertical displacement diagram with inclination $30^{\circ}$

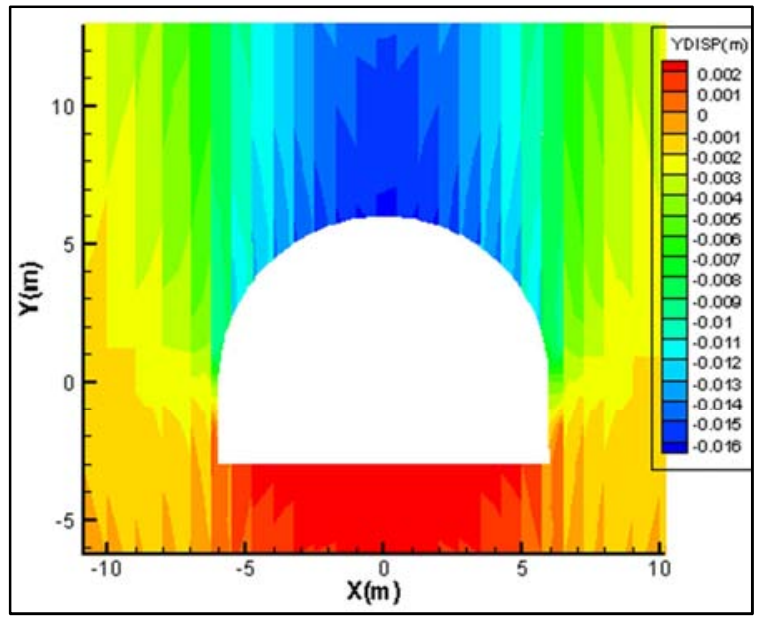

(c) Vertical displacement diagram with inclination $90^{\circ}$

Figure 3. Vertical displacement diagram of tunnel with different inclinations.

It can be seen from Figure. 3 that with the increase of the inclination angle of the structural plane, the phenomenon of abscission layers appears in the vertical displacement cloud diagrams. When the inclination angle of the structural plane is $30^{\circ}$, the phenomenon of abscission layers are obvious, and the inclination direction of these abscission layers are generally consistent with inclination angles direction of the structural plane. When the inclination angle of the structural surface is $0^{\circ}$ and $90^{\circ}$, the maximum vertical displacement occurs at the vault, and the vertical displacement cloud diagram of the rock mass presents a symmetrical distribution. When it is $30^{\circ}$, the maximum vertical displacement occurs at the arch waist, and the vertical displacement cloud chart of the rock mass presents an asymmetric distribution.

\subsubsection{Horizontal Displacement Analysis}

The numerical simulation of the horizontal displacement is the same as the vertical displacement. The calculation results of the maximum horizontal displacement of 28 working conditions are shown in Figure 4. 


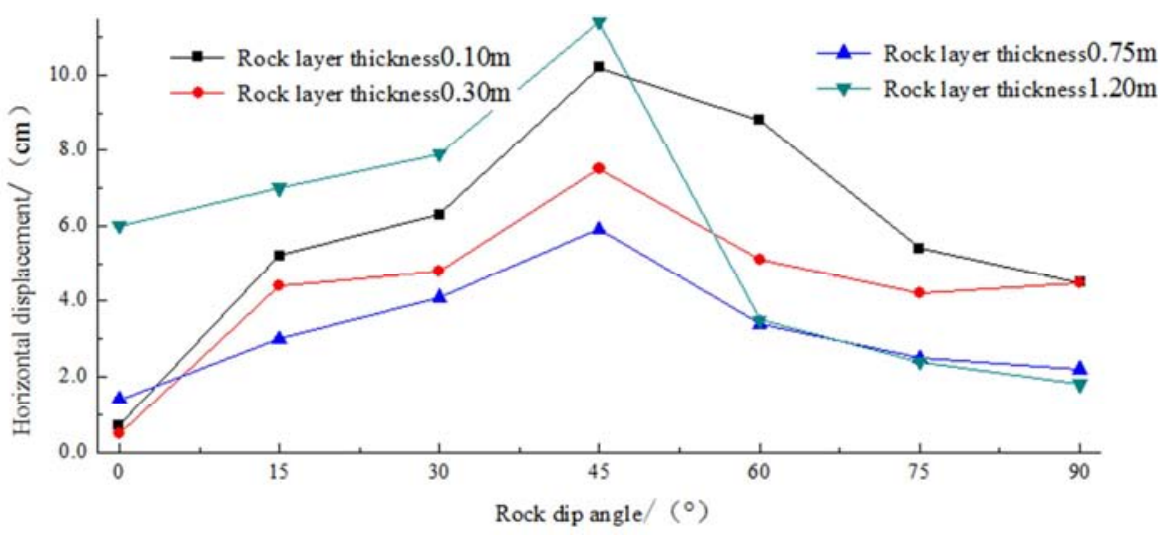

Figure 4. The maximum horizontal displacement corresponding to the different inclinations.

It can be seen from Figure 4 that when the inclination angle of the structural plane is between $0^{\circ}$ and $45^{\circ}$, the horizontal displacement of the surrounding rock of the layered surrounding rock tunnel increases with the increase of the inclination angle of the structural plane. When the inclination angle is $45^{\circ}$, the horizontal displacement of the surrounding rock of the layered surrounding rock tunnel reaches the maximum, which is $11.4 \mathrm{~cm}$. When the inclination angle of the structural plane is between $45^{\circ}$ and $90^{\circ}$, the horizontal displacement of the surrounding rock of the layered surrounding rock tunnel decreases with the increase of the inclination angle of the structure plane. When the inclination angle of the structural surface is $90^{\circ}$, the maximum horizontal displacement of surrounding rock of the layered surrounding rock tunnel decreases to $1.8 \mathrm{~cm}$. Therefore, the horizontal displacement of the surrounding rock of the layered surrounding rock tunnel are increasing first and then decreasing with the increase of the inclination angle of the structural plane. At the same time, there is a correlation between the thickness of rock layer and the horizontal displacement of surrounding rock. For the rock layers of $0.10 \mathrm{~m}, 0.30 \mathrm{~m}$ and $0.75 \mathrm{~m}$, when the inclination angle of the structural plane is between $15^{\circ}$ and $90^{\circ}$, the horizontal displacement of the surrounding rock of the layered surrounding rock tunnel decreases with the increase of the layer thickness. For the rock layers of $0.10 \mathrm{~m}, 0.30 \mathrm{~m}$, $0.75 \mathrm{~m}$ and $1.20 \mathrm{~m}$, when the inclination angle of the structural plane inclination is between $60^{\circ}$ and $90^{\circ}$, the horizontal displacement of the surrounding rock of the layered surrounding rock tunnel decreases with the increase of the layer thickness. Therefore, with the increase of rock layer thickness, the horizontal displacement of surrounding rock in layered surrounding rock tunnel is roughly reduced.

The same as the vertical displacement, from the calculation process, the position of the maximum horizontal displacements of the rock layers with different inclination angles also show a certain regularity. In order to reflect the change of horizontal displacement of different inclination angles, the rock layer thickness of $0.75 \mathrm{~m}$, the inclination angle of rock layers of $0^{\circ}, 45^{\circ}$ and $90^{\circ}$ are explained. The horizontal displacement cloud diagrams of different inclination angles are shown in Figure 5.
As can be seen from Figure 5, when the inclination angle of the structural plane is $0^{\circ}$, the maximum horizontal displacement appears at the left and right arch waists and the maximum horizontal displacement cloud diagram presents a symmetrical distribution. When the structural plane inclination angle is $45^{\circ}$, the horizontal displacement of the left arch waist and the right wall are large, but the maximum horizontal displacement appears at the left arch waist. When the structural plane inclination angle is $90^{\circ}$, the maximum horizontal displacement appears at the left and right side walls.

\subsection{Maximum Principal Stress Analysis}

When excavating a tunnel in the layered surrounding rock, the stress field around the tunnel will also affect its stability to some extent with the change of the inclination angle of the structure plane and the thickness of the rock layer. Therefore, numerical simulation was carried out at $0^{\circ}, 15^{\circ}, 30^{\circ}, 45^{\circ}, 60^{\circ}$, $75^{\circ}$ and $90^{\circ}$ as well as the thickness of $0.10 \mathrm{~m}, 0.30 \mathrm{~m}, 0.75$ $\mathrm{m}$ and $1.20 \mathrm{~m}$ respectively. The maximum principal stress calculated under 28 simulated conditions are shown in Figure 6.

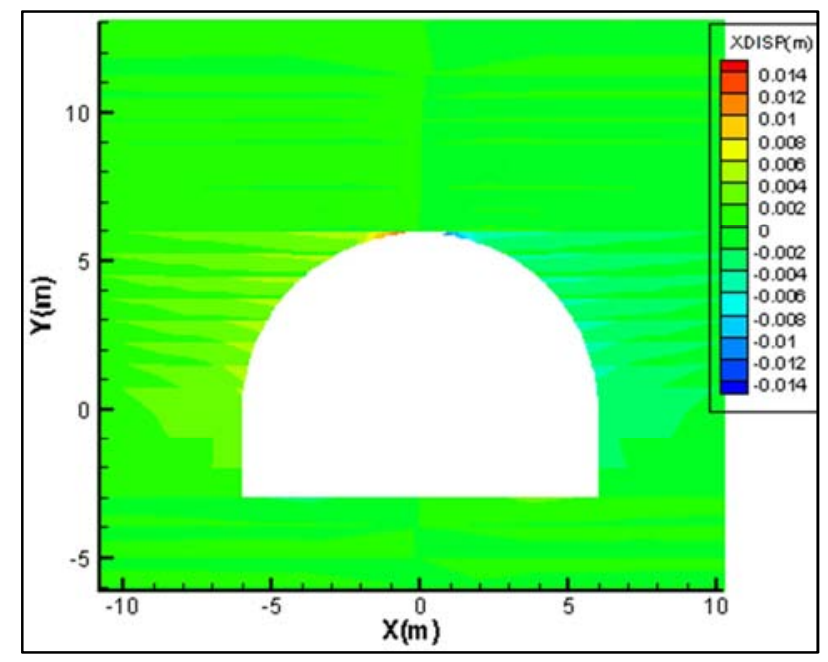

(a) Horizontal displacement diagram with inclination $0^{\circ}$. 


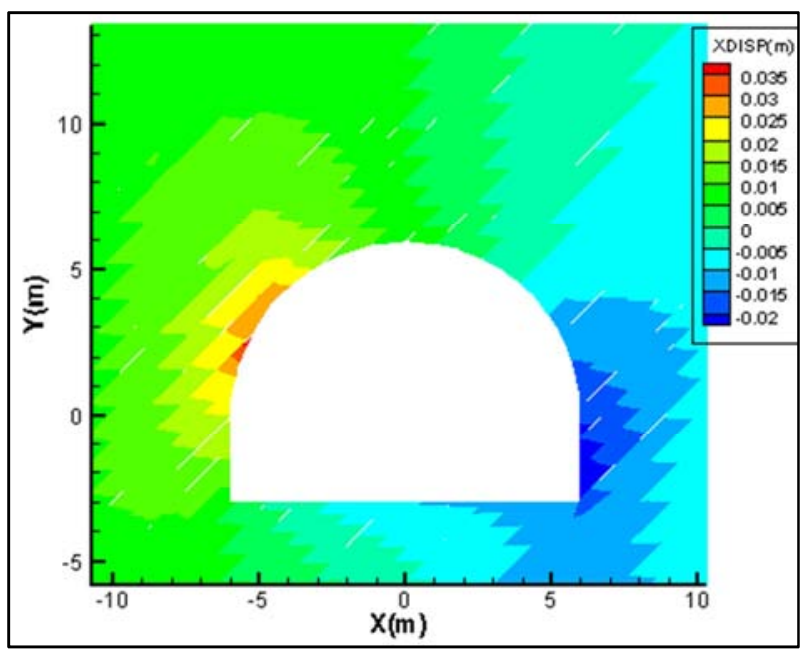

(b)Horizontal displacement diagram with inclination $45^{\circ}$.

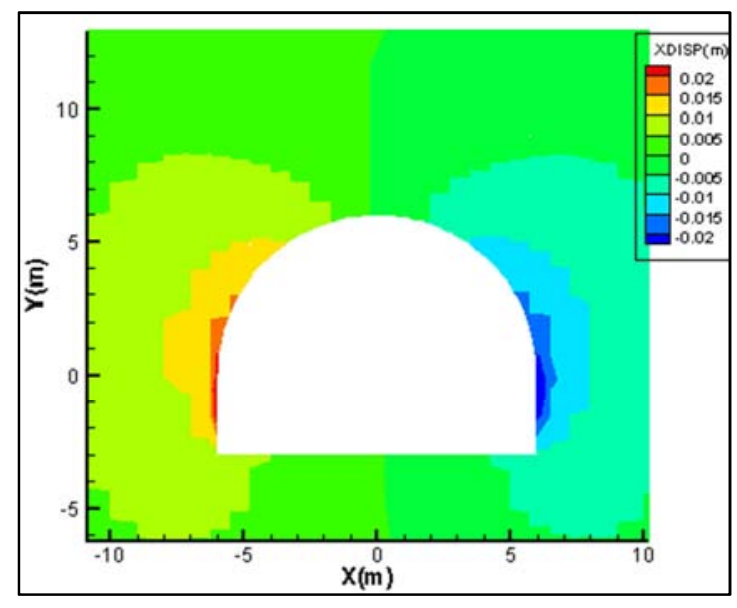

(c) Horizontal displacement diagram with inclination $90^{\circ}$.

Figure 5. Horizontal displacement diagram of tunnel with different inclinations.

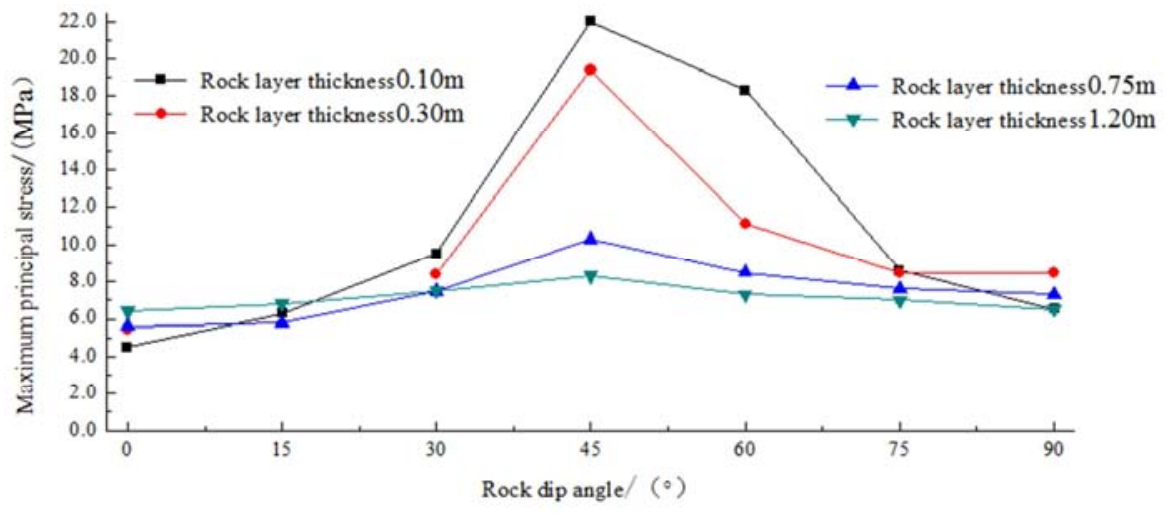

Figure 6. The maximum principal stress corresponding to the different inclinations.

As can be seen from Figure 6, when the inclination angle of the structural plane is between $0^{\circ}$ and $45^{\circ}$, the maximum principal stress of the layered surrounding rock tunnel rises with the increase of the inclination angle of the structural plane. When the inclination angle is $45^{\circ}$, the principal stress of the layered surrounding rock tunnel reaches the maximum, and the maximum value is $22.0 \mathrm{MPa}$. When the inclination angle of the structural plane is between $45^{\circ}$ and $90^{\circ}$, the maximum principal stress of the layered surrounding rock tunnel decreases with the increase of the inclination angle of the structural plane. When it is $90^{\circ}$, the maximum principal stress of the layered surrounding rock tunnel is reduced to 6.5 $\mathrm{MPa}$. Therefore, the maximum principal stress experienced by the layered surrounding rock tunnel increases first and then decreases with the increase of the inclination angle of the structural plane. When the layer thickness of the rock layer is $0.10 \mathrm{~m}$, the maximum principal stress of the layered surrounding rock tunnel is $22.0 \mathrm{MPa}$. When the layer thickness of the rock layer is $0.30 \mathrm{~m}$, the maximum principal stress of the layered surrounding rock tunnel is $19.4 \mathrm{MPa}$. When it is $0.75 \mathrm{~m}$, the maximum principal stress of the layered surrounding rock tunnel is $10.3 \mathrm{MPa}$. When the layer thickness is $1.20 \mathrm{~m}$, the maximum principal stress of the layered surrounding rock tunnel is $8.3 \mathrm{MPa}$, as compared with the layer thickness of $0.10 \mathrm{~m}$ formation is reduced 2.6 times. Because of the small dispersion in the small angle of inclination, the maximum principal stress of the layered surrounding rock tunnel is roughly decreasing with the increase of the rock layer thickness. At the same time, for the layer thickness of $0.10 \mathrm{~m}$ and $0.30 \mathrm{~m}$, when the inclination angle is substantially $30^{\circ}$ and $75^{\circ}$, a change rate of the maximum principal stress layer rock tunnel being faster.

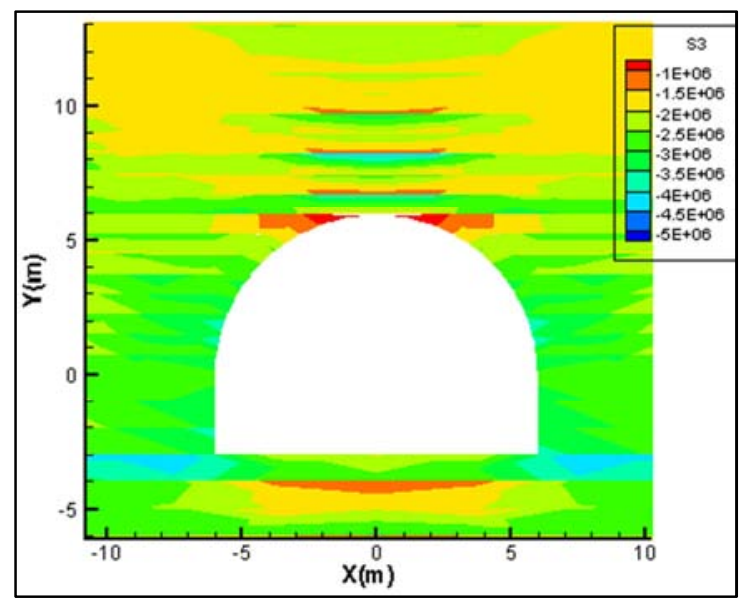

(a) Maximum principal stress diagram with inclination $0^{\circ}$. 


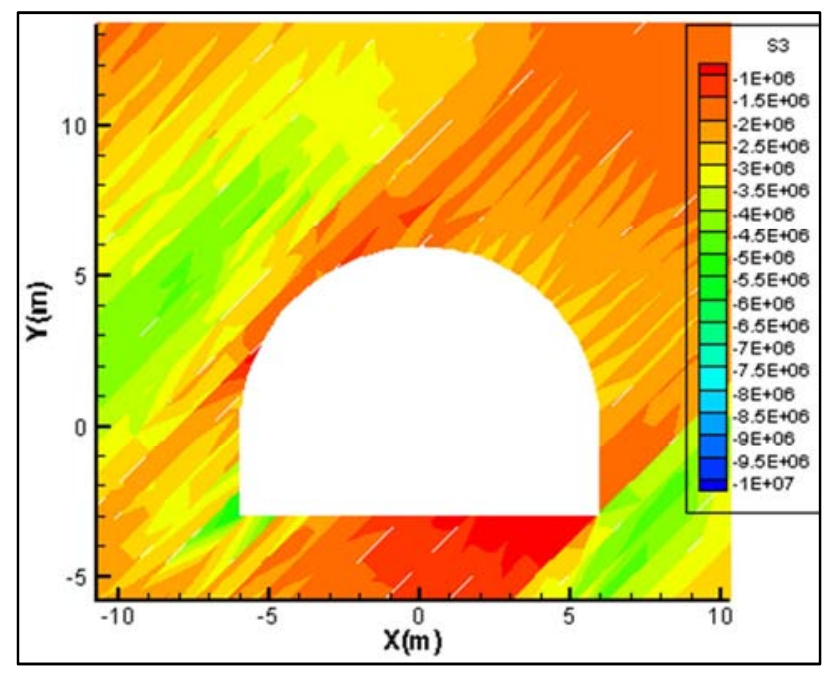

(b) Maximum principal stress diagram with inclination $45^{\circ}$.

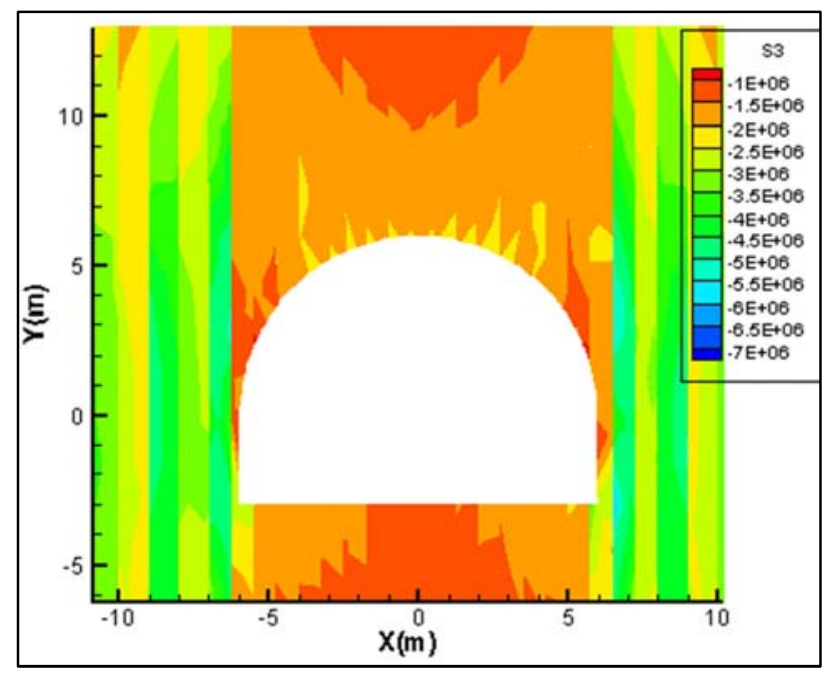

(c) Maximum principal stress diagram with inclination $90^{\circ}$.

Figure 7. Maximum principal stress diagram of tunnel with different inclinations.

From the calculation process, the cloud diagrams of the maximum principal stress of rock formations with different inclination angles also shows certain regularity. In this paper, the layer thickness of $0.75 \mathrm{~m}$, the inclination angle of rock layers of $0^{\circ}, 45^{\circ}$ and $90^{\circ}$ are explained. The maximum principal stress cloud diagrams for different inclination angles are shown in Figure 7.

As can be seen from Figure 7, when the inclination angle of the structural plane is $0^{\circ}$ and $90^{\circ}$, the maximum principal stress cloud diagram of the rock mass presents a symmetrical distribution. When the inclination angle of the structural plane is $45^{\circ}$, the cloud diagram of the maximum principal stress of the rock mass presents an asymmetric distribution and even comes into being eccentric-pressed tunnel.

\section{Conclusion}

Since the stability of the surrounding rock after the excavation of the layered surrounding rock tunnel is closely related to the layer thickness and inclination angle of the layered rock mass, the inclination angles of the rock layers are $0^{\circ}, 15^{\circ}, 30^{\circ}, 45^{\circ}, 60^{\circ}, 75^{\circ}$ and $90^{\circ}$ respectively. For the case of layer thicknesses of $0.10 \mathrm{~m}, 0.30 \mathrm{~m}, 0.75 \mathrm{~m}$ and 1.20 $\mathrm{m}$, the discrete element software is used for numerical simulation, and the effects of different inclination angles and thicknesses on vertical displacement, horizontal displacement and maximum principal stress of surrounding rock are analyzed. The main conclusions are the following points:

(1) Under the condition of the same layer thickness and excavation span, the vertical displacement of the surrounding rock of the layered surrounding rock tunnel are increasing first and then decreasing with the increase of the inclination angle of the structural plane. When the inclination angle is $30^{\circ}$, the vertical displacement of surrounding rock of the layered surrounding rock tunnel appears the maximum value, which is $9.9 \mathrm{~cm}$. Under the same inclination angle and excavation span, the vertical displacement of the surrounding rock gradually decreases with the increase of layer thickness.

(2) Under the condition of the same layer thickness and excavation span, the horizontal displacement of the surrounding rock of the layered surrounding rock tunnel are increasing first and then decreasing with the increase of the inclination angle of the structural plane. When the inclination angle is $45^{\circ}$, the horizontal displacement of surrounding rock of the layered surrounding rock tunnel appears the maximum value, which is $11.4 \mathrm{~cm}$. Under the same inclination angle and excavation span, the horizontal displacement of the surrounding rock gradually decreases with the increase of layer thickness.

(3) Under the condition of the same layer thickness and excavation span, the maximum principal stress of the surrounding rock of the layered surrounding rock tunnel are increasing first and then decreasing with the increase of the inclination angle of the structural plane. When the inclination angle is $45^{\circ}$, the maximum principal stress of surrounding rock of the layered surrounding rock tunnel appears the maximum value, which is $22.0 \mathrm{MPa}$. Under the condition of the same inclination angle and excavation span, the maximum principal stress of the layered surrounding rock tunnel decreases with the increase of the layer thickness.

\section{Acknowledgements}

This research was financially supported by the National Natural Science Foundation of China (51408054); the Natural Science Basic Foundation by Shaanxi Province (2017JM5136); the Scientific Research Program for Technology of Highway Construction and Maintenance Technology of National Transportation Industry Key Laboratory(KLTLR-Y14-15); the Housing and Urban-Rural Construction Foundation by the Housing and Urban-Rural Department of Shaanxi Province (2017-K55); the Special Scientific Research Program of Education Department of Shaanxi Province (18JK0402). 


\section{References}

[1] Tan xin, Fu helin, Chen chen, Zhao minghua, Liu yunsi (2016). Numerical simulation analysis of tunnel in layered rock mass. Journal of Railway Science and Engineering 13 (06), 1108-1113.

[2] Liu Hongbing (2013). Analysis of impact on tunnels stability with layered rocks. Highway Engineering 38 (04), 167-169+182.

[3] Xia binwei, Chen guo, Kang yong, Zhou dongping (2010). Deformation characteristic and stability analyses of layered rockmass. Hydrogeology and Engineering Geology 37 (04), 48-52.

[4] Geng (2003). Daxin, Yang Linde. Mechanical characteristics of layered rock mass and numerical modeling. Underground Space 23 (04), 380-387. (in Chinese)

[5] Yang Chaofeng (2018). Stability Analysis of Tunnel Surrounding Rock Mass in Layered Rock Based on Discrete Element Method. Journal of Luoyang Institute of Science and Technology (Natural Science Edition) 28 (02), 28-32.

[6] Zhou Kefeng, Li Yuzhi, Liu Junyi (2012). Numerical analysis of structure plane characteristic for strength of stratified rock mass. Journal of Central South University (Science and Technology) 43 (04), 1424-1428.

[7] Jia Peng, Tang Chunan, Yang Tianhong, Wang Shuhong (2006). Numerical stability analysis of surrounding rock mass layered by structural planes with different obliquities. Journal of Northeastern University (Natural Science) (11), 1275-1278.

[8] Zhao Jingeng (2011). Study on stability of large-section tunnel of layered rock mass by joint inclination angle. Railway Engineering (09), 58-61.
[9] Song Chengke, Wang Chenghu, Huang Luyuan, et al (2012) Numerical Simulation of Influence of Structural Plane Distribution Characteristic on Deformation of Tunnel's Surrounding Rock. Journal of Disaster Prevention and Mitigation Engineering 32 (5), 611.(in Chinese)

[10] Ou Erfeng, Ma Xuening, Yan Songhong (2018). Research on the Seismic Response Characteristics of Railway Tunnel in Horizontal Layered Surrounding Rock. Journal of Railway Engineering Society 35 (05), 88-93.

[11] Wang Yonggang, Ding Wenqi, Jia Shanpo, Zou Chensong (2017). Anisotropic model of layered rock mass considering characteristics of structual interface. Journal of Highway and Transportation Research and Development 31 (10), 85-92.

[12] Wang Linfeng, Tang Hongmei, Tang Fen, Ye Siqiao (2017). Failure mechanism of gently inclined bedding rock mass slopes with complex fissures. Chinese Journal of Geotechnical Engineering 39 (12), 2253-2260.

[13] Wang Hui, Yang Shuangsuo, Niu Shaoqing (2016). Deformation Mechanism of Roadway with Layered Compound Strata and Its Control. Technology. Journal of Taiyuan University of Technology 47 (05), 605-612.

[14] JTGD70/2-2014. The Code for Design of Road Tunnel, Chinese standard, China, 2014.

[15] Z. X. Zhang, Y. Xu, P. H. S. W. Kulatilake et al (2012). Physical Model Test and Numerical Analysis on the Behavior of Stratified Rock Masses during Underground Excavation. International Journal of Rock Mechanics and Mining Sciences 49 (49). 